\title{
T1 invasive lung adenocarcinoma: Thin-section CT solid score and histological periostin expression predict tumor recurrence
}

\author{
RYOJI IWAMOTO ${ }^{1}$, SHUICHI TANOUE ${ }^{1}$, SHUJI NAGATA ${ }^{1}$, KAZUHIRO TABATA ${ }^{2}$, JUNYA FUKUOKA ${ }^{2}$, \\ MASAMICHI KOGANEMARU ${ }^{1}$, AKIKO SUMI ${ }^{1}$, TOMONORI CHIKASUE ${ }^{1}$, TOSHI ABE $^{1}$, DAIGO MURAKAMI ${ }^{3}$, \\ SHINZO TAKAMORI ${ }^{3}$, HIDENOBU ISHII ${ }^{4}$, KOICHI OHSHIMA ${ }^{5}$, SHOICHIRO OHTA $^{6}$, \\ KENJI IZUHARA $^{6}$ and KIMINORI FUJIMOTO ${ }^{1}$ \\ ${ }^{1}$ Department of Radiology, Kurume University School of Medicine, Kurume, Fukuoka 830-0011; \\ ${ }^{2}$ Department of Pathology, Nagasaki Graduate School of Biomedical Sciences, Nagasaki 852-8523; \\ ${ }^{3}$ Department of Surgery; ${ }^{4}$ Division of Respirology, Neurology, and Rheumatology, Department of Internal Medicine; \\ ${ }^{5}$ Department of Pathology, Kurume University School of Medicine, Kurume, Fukuoka 830-0011; \\ ${ }^{6}$ Division of Medical Biochemistry, Department of Biomolecular Sciences, Saga Medical School, Saga 849-8501, Japan
}

Received March 15, 2021; Accepted August 12, 2021

DOI: $10.3892 / \mathrm{mco} .2021 .2391$

\begin{abstract}
Adenocarcinoma is the most common histological type of non-small cell lung cancer (NSCLC), and various biomarkers for predicting its prognosis after surgical resection have been suggested, particularly in early-stage lung adenocarcinoma. Periostin (also referred to as POSTN, PN or osteoblast-specific factor) is an extracellular matrix protein, the expression of which is associated with tumor invasiveness in patients with NSCLC. In the present study, the novel approach, in which the thin-section CT findings prior to surgical resection and periostin expression of resected specimens were analyzed in combination, was undertaken to assess whether the findings could be a biomarker for predicting the outcomes following resection of $\mathrm{T} 1$ invasive lung adenocarcinoma. A total of 73 patients who underwent surgical resection between January 2000 and December 2009 were enrolled. A total of seven parameters were assessed in the thin-section CT scans: i) Contour; ii) part-solid ground-glass nodule or solid nodule; iii) percentage of solid component (the CT solid score); iv) presence of air-bronchogram and/or bubble-like lucencies; v) number of involved vessels; vi) shape linear strands between the nodule and the visceral pleura; and vii) number of linear strands between the nodule and the visceral pleura. Two chest radiologists independently assessed the parameters. Periostin expression was evaluated on the basis of the strength and extent of staining. Univariate and multivariate analyses were
\end{abstract}

Correspondence to: Professor Kiminori Fujimoto, Department of Radiology, Kurume University School of Medicine, 67 Asahi-machi, Kurume, Fukuoka 830-0011, Japan

E-mail: kimichan@med.kurume-u.ac.jp

Key words: lung adenocarcinoma, multidetector computed tomography, solid component, periostin protein, prognosis subsequently performed using the Cox proportional hazards model. There was a substantial to almost perfect agreement between the two observers with regard to classification of the seven thin-section CT parameters $(\kappa=0.64-0.85)$. In the univariate analysis, a CT solid score $>80 \%$, pathological lymphatic invasion, tumor and lymph node status and high periostin expression were significantly associated with recurrence (all $\mathrm{P}<0.05$ ). Multivariate analysis demonstrated that a CT solid score $>80 \%$ and high periostin expression were risk factors for recurrence $(\mathrm{P}=0.002$ and $\mathrm{P}=0.011$, respectively). The cumulative recurrence rates among the three groups (both negative, CT solid score $>80 \%$ or high periostin expression, or both positive) were significantly different (log-rank test, $\mathrm{P}<0.001)$. Although the solid component is already known to be a major predictor of outcome in lung adenocarcinomas according to previous studies, the combined analysis of CT solid score and periostin expression might predict the likelihood of tumor recurrence more precisely.

\section{Introduction}

Adenocarcinoma is the most common histological type of nonsmall cell lung cancer (NSCLC) (1). In 2011, a new histological typing for lung adenocarcinoma was established in the consensus classification by the International Association for the Study of Lung Cancer/American Thoracic Society/European Respiratory Society (IASLC/ATS/ERS) (2). This classification includes three main subtypes: Adenocarcinoma in situ (AIS), minimally invasive adenocarcinoma (MIA) and invasive adenocarcinoma. Invasive adenocarcinoma consists of five patterns, according to the predominant histological type (lepidic, papillary, acinar, solid or micropapillary pattern) (2). The prognosis of AIS and MIA is favorable, with a 5-year survival rate of $100 \%$ for both tumor types following surgical resection. However, invasive adenocarcinoma, excluding the lepidic predominant pattern, has a relatively less favorable prognosis compared with AIS and MIA, even in the case of 
smaller tumors (3-5). Lee et al (4) reported that the 5-year disease-free survival rate for invasive adenocarcinoma with a maximum tumor size $\leq 3 \mathrm{~cm}$ after surgical resection varied from $50-93 \%$ according to the histological pattern. Kadota et al (5) reviewed tumor slides with pathological stage I lung adenocarcinoma (TNM classification, 7th edition) (6), and showed that the 5-year cumulative incidence of recurrence was $0 \%$ in patients with AIS and MIA, $8 \%$ in lepidic predominant invasive adenocarcinoma and $19 \%$ in non-lepidic predominant invasive adenocarcinoma. The patients after surgical resection of early-stage lung adenocarcinoma are commonly follow-up without adjuvant therapy. But they also contain some worse prognostic cases. Therefore, various biomarkers for predicting the prognosis of patients following surgical resection have been suggested, particularly for early-stage lung adenocarcinoma $(4,7,8)$. It is important that the patients after operation are classified in personalized follow-up schedule according to prognostic biomarker even in earlier stage.

Periostin is an extracellular matrix protein initially identified as OSF-2 in the mouse MC3T3-E1 osteoblastic cell line (9). In humans, the protein is composed of $\sim 800$ amino acids, having a molecular weight of $90 \mathrm{kDa}(10)$. As a matricellular protein, periostin has defined functions in osteology, tissue repair, oncology, the cardiovascular and respiratory systems, as well as in various inflammatory settings and diseases $(11,12)$. In oncology, periostin upregulation has been observed in various types of neoplasms (13-18), and the protein is hypothesized to serve a role in invasion, angiogenesis and metastasis in vitro and in vivo (13). In patients with NSCLC, periostin expression is associated with overall survival and/or tumor invasiveness $(15,19)$; however, previous studies on NSCLC have included patients of various stages and histological types, and the correlation between periostin expression and the prognosis of lung adenocarcinoma at earlier stages has not been assessed, to the best of our knowledge.

As an imaging technique commonly used for NSCLC, several studies have reported an association between pre-operative computed tomography (CT) findings and postoperative prognosis (20-24). These studies all reported that a large degree of ground-glass opacity (GGO) on a pre-operative CT scan is indicative of a favorable prognosis, whereas a large solid component is associated with a poorer outcome. However, one disadvantage was that the patients included in these studies had varying degrees of tumor invasiveness, tumor stages and histological types.

In an attempt to shed further light on this issue, in the present study small lung adenocarcinomas (excluding AIS and MIA) were examined. The cases were limited to those of T1 adenocarcinoma (TNM classification, 8th edition) (25) that were also pathologically diagnosed as invasive adenocarcinoma according to the present IASLC/ATS/ERS histological classification (2). To the best of our knowledge, there are no previous reports comprehensively evaluating the prognosis using both imaging characteristics and histological features, including an analysis of the periostin expression level in patients with NSCLC. In the present study, the value of thin-section CT findings and histological features, including periostin immunostaining, in predicting outcomes in patients after resection of $\mathrm{T} 1$ invasive lung adenocarcinoma were determined.

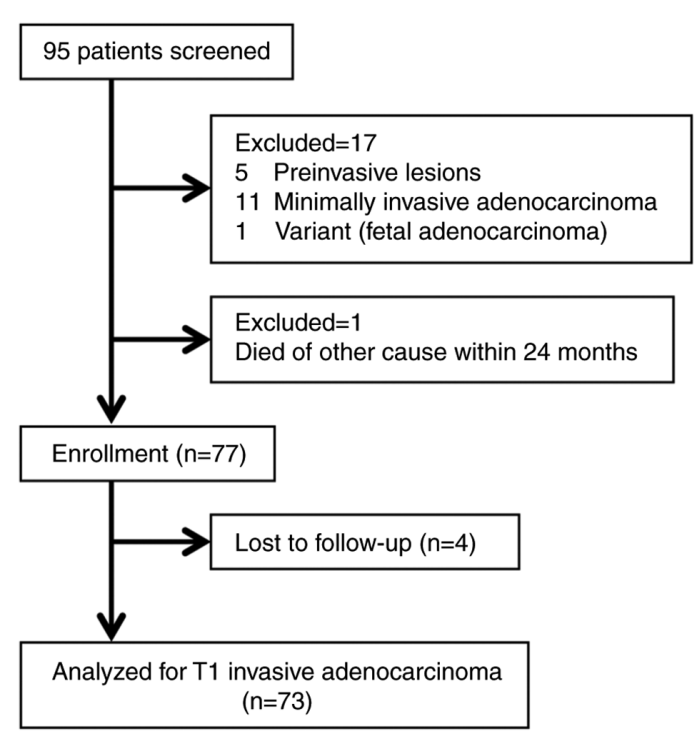

Figure 1. Diagram of patient selection.

\section{Patients and methods}

Study subjects. The Ethics Committee of Kurume University (approval no. 09113) approved this retrospective study, and waived the requirement for informed consent. A total of 95 consecutive patients were included in the present study who met the following inclusion criteria when retrospectively screened: i) surgical resection was performed at Kurume University Hospital between January 2000 and December 2009 with a chest thin-section CT scan within the 4 weeks prior to surgery; ii) a histopathological diagnosis of invasive adenocarcinoma was made with a solid component measuring $\leq 3 \mathrm{~cm}$ on the thin-section CT scan; and iii) no invasion of the visceral pleura was observed upon histopathological analysis. Of the 95 consecutive patients who met the inclusion criteria, 22 were excluded from the study: 17 were excluded due to inadequate pathological findings, 4 patients were lost to follow-up, and 1 patient died of a cause other than cancer within 24 months following surgery. Ultimately, a total of 73 patients (39 men and 34 women) were included (Fig. 1). The basic operative procedure was a lobectomy with regional mediastinal and hilar lymph node dissection (26). None of the recruited patients had received any pre-operative therapies.

Postoperative adjuvant therapy, including chemotherapy, radiation or other therapies, were performed for the patients in accordance with established criteria.

CT scans and interpretation of images. Pre-operative CT was performed with a variety of scanners (16-, 64- and 128-channel multidetectors). All CT examinations were obtained from the lung apices to the middle portion of both kidneys on full inspiration whilst the patient was in the supine position. Scanning parameters were as follows: Kilovoltage peak $(\mathrm{kVp})$ and current, $120 \mathrm{kVp}$ and 150-450 mA, respectively; rotation, 0.35-0.5 sec; pitch, 0.984-0.992:1; section thickness, $1.25 \mathrm{~mm}$; matrix, 512x512; and field of view, $35 \mathrm{~cm}$. These imaging data were reconstructed with a high spatial frequency algorithm at a thickness of $1.25 \mathrm{~mm}$. All images were interpreted using a window width of 1,500-1,600 Hounsfield units (HU) and a window level of -600 to $-700 \mathrm{HU}$ for the lung window settings, 
A
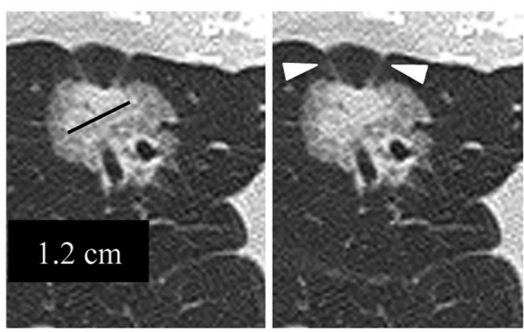

C

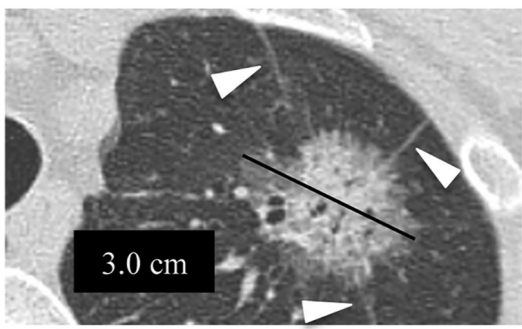

E

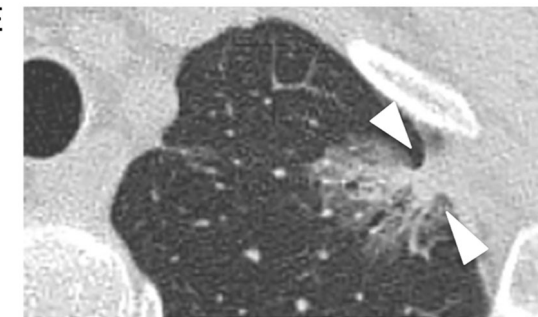

G

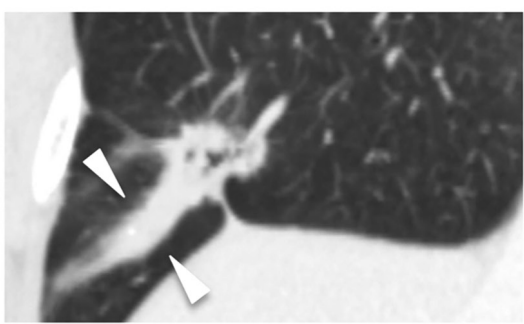

B

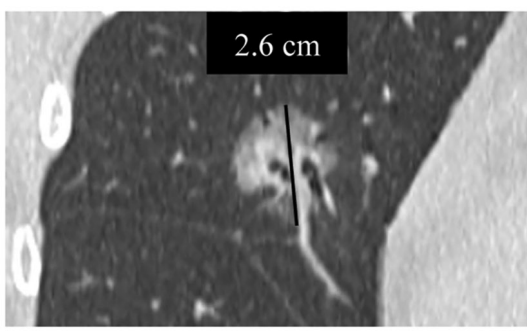

D

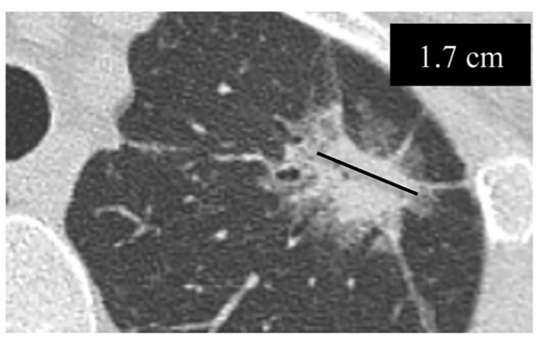

F

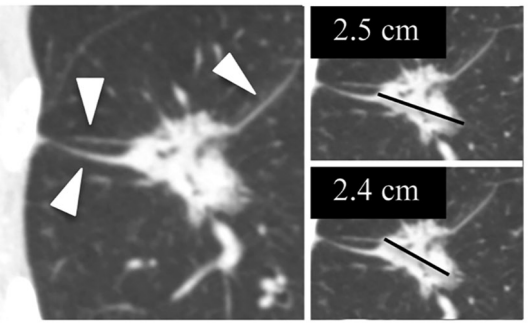

$\mathrm{H}$

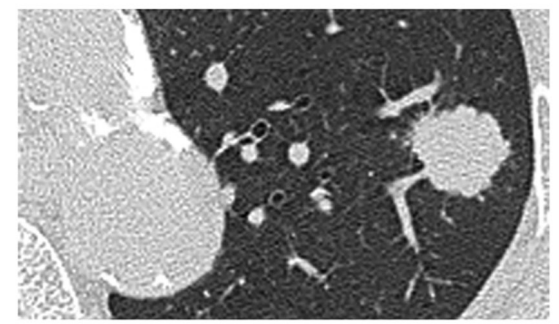

Figure 2. Examples of thin-section CT image parameters. (A) A part-solid GGN in a 63-year-old woman located at the right middle lobe, showing smooth contour, pathologically diagnosed as invasive adenocarcinoma (papillary predominant). The maximal diameter of the solid component was obtained at axial image. The black line indicates the traced maximal diameter of the solid part, which was measured as $1.2 \mathrm{~cm}$. The shape of the linear strand between the nodule and the visceral pleura (number, $\geq 2$ ) was fine (arrow heads). (B) Coronal image using MPR of the same patient as in (A). The maximal diameter of the whole nodule was obtained at coronal image. The black line indicates the traced maximal diameter, which was measured as $2.6 \mathrm{~cm}$. The CT solid score was calculated as $46 \%$ (solid part, $1.2 \mathrm{~cm} / \mathrm{whole}$ nodule, $2.6 \mathrm{~cm}$ ). (C) A part-solid GGN in a 62-year-old woman located at the left upper lobe, showing irregular contour and air-bronchogram and/or bubble-like lucencies, pathologically diagnosed as invasive adenocarcinoma (lepidic predominant). The maximal diameter of the whole nodule was obtained at axial image. The black line indicates the traced maximal diameter measured as $3 \mathrm{~cm}$. The number of linear strands between the nodule and the visceral pleura was 3 (arrow heads). (D) Different axial section of the same patient as in (C). The maximal diameter of the solid component was also obtained at axial image. The black line indicates the traced maximal diameter of the solid part measured as $1.7 \mathrm{~cm}$. The CT solid score was calculated as $57 \%$ (solid part, $1.7 \mathrm{~cm} /$ whole nodule, $3.0 \mathrm{~cm}$ ). (E) Different axial section of the same patient as in (C and D). The shape of the linear strand between the nodule and the visceral pleura was triangular (arrow heads), which was categorized as type 2 (coarse or triangular). (F) A part-solid GGN in a 66-year-old man located at the right lower lobe, showing irregular contour and air-bronchogram and/or bubble-like lucencies, pathologically diagnosed as invasive adenocarcinoma (papillary predominant). The maximal diameters of solid component and the whole nodule were obtained at the same axial section. The black line in the right upper image indicates the traced maximal diameter of the whole nodule measured as $2.5 \mathrm{~cm}$, and the line in the right lower image indicates the traced maximal diameter of the solid part measured as $2.4 \mathrm{~cm}$. The CT solid score was calculated as $92 \%$ (solid part, $2.4 \mathrm{~cm} /$ whole nodule, $2.5 \mathrm{~cm}$ ). The number of linear strands between the nodule and the visceral pleura was 3 (arrow heads). (G) Coronal image using MPR of the same patient as in (F). The shape of the linear strand between the nodule and the visceral pleura was coarse (arrow heads), which was categorized as type 2.(H) A solid nodule without GGO in a 78-year-old man located at left upper lobe, showing irregular contour, pathologically diagnosed as invasive adenocarcinoma (acinar predominant). The maximal diameter of the solid component corresponded to the whole nodule, measuring $2.0 \mathrm{~cm}$ (CT solid score, 100\%). Linear strands between the nodule and the visceral pleura were not observed. GGN, ground-glass nodule; MPR, multiplanar reformation; GGO, ground-glass opacity.

and a window width of 350-400 HU and a window level of 30-40 HU for the mediastinal window settings (27).

The CT findings were assessed independently by two board-certified chest radiologists (with 17 and 31 years of experience, respectively) without knowledge of any clinical or histological findings, with the exception that all pulmonary nodules had been pathologically diagnosed as primary lung adenocarcinoma. When the results from the two observers differed, final decisions were reached by consensus after calculation of the independent interobserver agreement ( $\kappa$ statistic). Morphological findings on thin-section CT for small invasive adenocarcinomas were evaluated for the following seven parameters: i) contour; ii) part-solid ground-glass nodule (GGN) or solid nodule; iii) percentage of the solid component (CT solid score); iv) presence of air-bronchogram and/or bubble-like lucencies; v) number of involved vessels in the tumor; and the vi) shape and vii) number of linear strands between the nodule and the visceral pleura (Fig. 2). Tumor contour was characterized 

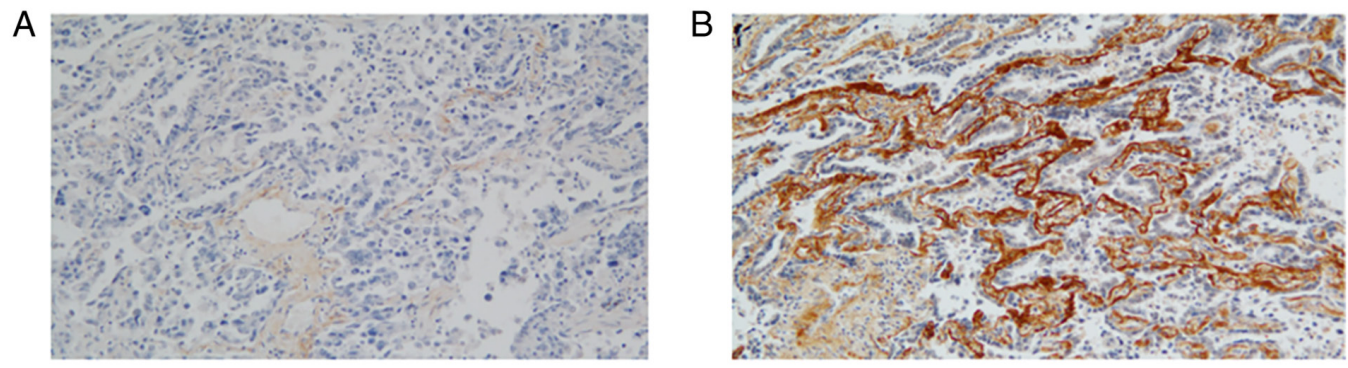

Figure 3. Examples of immunohistochemical staining for periostin. (A) Periostin immunohistochemistry of lung adenocarcinoma (original magnification, x100) The strength of staining was evaluated as weak (1 point) and the extent of staining was evaluated as $<50 \%$ ( 1 point). Therefore, total score was 2 , and periostin expression was defined as low. (B) Periostin immunohistochemistry of lung adenocarcinoma (magnification, x100). The strength of staining was estimated as strong ( 3 points) and the extent of staining was evaluated as $>50 \%$ ( 2 points). Therefore, the total score was 5 , and periostin expression was defined as high.

as being either smooth or irregular. For the evaluation of tumor size, the longest diameters of the solid component and of the whole nodule, including GGO, were measured. Measurements were performed following multiplanar reformation in the plane showing the longest diameters. The CT solid score was calculated by dividing the longest diameter of the solid component by the longest diameter of the whole nodule, and multiplying by 100 . The subjects were divided into two groups based on the CT solid score to evaluate the association between the prognosis and solid scoring. The threshold of solid score for the grouping was calculated using receiver operating characteristic (ROC) curve analysis to obtain the highest sensitivity and specificity for predicting recurrence and death. The criteria for air-bronchogram and/or bubble-like lucencies were the same as those described previously (28-30). Concerning the linear strands between the nodule and visceral pleura, it was hypothesized that it would be possible to discern central fibrotic changes of the tumor. Linear strands were divided into two groups according to the morphological feature on CT: Type 1, none or fine; and type 2, coarse or triangular (Fig. 2). If the lesion had linear strands of both fine shape and coarse or triangular shape, it was categorized as type 2. Involved vessels were considered as all those abutting the nodule, and this was stratified on multiplanar reformations as being 2 vessels and over or less. Recurrence on follow-up CT included both local recurrence and distant metastases.

Histopathological and immunohistochemical staining and assessment. Resected lung tissues were fixed with formaldehyde, and paraffin-embedded tissue sections were obtained. All cases were classified according to the current TNM classification for lung cancer described in the IASLC/ATS/ERS histological classification (2), and the IASLC staging classification (8th edition) (25) by the consensus of two experienced pathologists (with 16 and 22 years of experience). Invasive adenocarcinomas were classified into one of five predominant types: Lepidic predominant, acinar predominant, papillary predominant, micropapillary predominant or solid predominant with mucin production, and invasive mucinous adenocarcinoma. Pathological T status, N status, lymphatic invasion and vessel invasion were also assessed.

The procedure of immunohistochemical staining for periostin was performed as previously described $(11,16)$. Tissue specimens were deparaffinized with xylene and serial dilutions of ethanol, and then were washed three times for 5 min in PBS (composition: pH 7.4, $130 \mathrm{mmol} / 1 \mathrm{NaCl}, 2 \mathrm{mmol} / 1 \mathrm{NaH}_{2} \mathrm{PO}_{4}$ and $7 \mathrm{mmol} / \mathrm{I} \mathrm{Na}_{2} \mathrm{HPO}_{4}$ ) and then blocked with $10 \%$ skimmed milk in PBS at room temperature for $30 \mathrm{~min}$. Sections were incubated overnight at $4^{\circ} \mathrm{C}$ with an originally developed rat anti-human periostin monoclonal antibody (SS19B or SS5D, diluted 1:100 in PBS). After several washes with PBS, the sections were incubated with the secondary antibody, namely biotin-labeled goat anti-rat IgG (cat. no. \#PK-4004; Vectastain ABC kit, Vector Laboratories, Inc.) diluted 1:100 in PBS at room temperature for $1 \mathrm{~h}$. The sections were washed with PBS again, positive reactivity was identified using a development reagent (Vectastain $\mathrm{ABC}$ kit) and the section were then visualized with 3,3'-diaminobenzidine (Dako; Agilent Technologies, Inc.).

Periostin expression was evaluated on the basis of the strength and extent of staining by an experienced pathologist (33 years of experience) who was not one of the two pathologists mentioned above, and who did not have knowledge of any other clinical data or the CT findings. The strength was evaluated at the strongest area of expression within the tumor, and was graded on a 4-point scale: 0 , no stain; 1 , weak; 2, moderate; or 3 , strong. The extent of staining was graded on a 3-point scale: $0,0 \% ; 1,<50 \%$; or $2, \geq 50 \%$. When the total score (sum of the strength and extent of staining) was $\geq 4$, periostin expression was defined as high, whereas a score $<4$ was defined as low (Fig. 3) (17,18).

Statistical analysis. Interobserver agreements on thin-section CT parameters were assessed using the $\kappa$ statistic, defined as follows: Poor, $\kappa<0.0$; slight, $\kappa=0.0-0.20$; fair, $\kappa=0.21-0.40$; moderate, $\kappa=0.41-0.60$; substantial, $\kappa=0.61-0.80$; or almost perfect, $\kappa=0.81-1.00$ (31). Survival was calculated from the date of surgery to the date of death or last contact. In the latter group (patients with the date of death unknown), survival was calculated using a right censoring survival model. Survival outcomes were obtained either from the patients' medical records or from the records of their primary care physicians or surgeons.

Associations between recurrence or overall survival (OS) and patient characteristics, pathological findings or CT parameters were analyzed using a $\chi^{2}$ or Fisher's exact test, as appropriate. Statistical differences for recurrence and OS according to the patient's age at surgery ( $>65$ or $\leq 65$ years), sex, IASLC/ATS/ERS histological typing, pathological lymphatic invasion, pathological vessel invasion, pathological $\mathrm{T}$ status, $\mathrm{N}$ status, each of the CT parameters described above, and periostin expression were analyzed using univariate and 
Table I. Comparison of patient characteristics and pathological findings between the no recurrence $(n=46)$ and recurrence $(n=27)$ groups, and between the survival $(\mathrm{n}=61)$ and death $(\mathrm{n}=12)$ groups.

\begin{tabular}{|c|c|c|c|c|c|c|}
\hline Categories & $\begin{array}{c}\text { No recurrence, } \\
\mathrm{n}(\%)\end{array}$ & $\begin{array}{c}\text { Recurrence, } \\
\mathrm{n}(\%)\end{array}$ & P-value & $\begin{array}{c}\text { Survival, } \mathrm{n} \\
(\%)\end{array}$ & $\begin{array}{l}\text { Death, } \\
\text { n }(\%)\end{array}$ & P-value ${ }^{a}$ \\
\hline Age at surgery, years & & & 0.057 & & & 0.221 \\
\hline$\leq 65$ & $18(39)$ & $17(63)$ & & $27(44)$ & $8(67)$ & \\
\hline$>65$ & $28(61)$ & $10(37)$ & & $34(56)$ & $4(33)$ & \\
\hline Sex & & & 0.812 & & & $>0.999$ \\
\hline Male & $24(52)$ & $15(56)$ & & $33(54)$ & $6(50)$ & \\
\hline Female & $22(48)$ & $12(44)$ & & $28(46)$ & $6(50)$ & \\
\hline WHO typing & & & 0.003 & & & 0.089 \\
\hline Lepidic predominant & $4(9)$ & $3(11)$ & & $5(8)$ & $2(17)$ & \\
\hline Acinar predominant & $3(6)$ & $11(41)$ & & $11(18)$ & $3(25)$ & \\
\hline Papillary predominant & $28(61)$ & $6(22)$ & & $32(53)$ & $2(17)$ & \\
\hline Micropapillary predominant & $4(9)$ & $2(7)$ & & $4(7)$ & $2(17)$ & \\
\hline Solid predominant with mucin production & $3(6)$ & $4(15)$ & & $4(7)$ & $3(25)$ & \\
\hline Invasive mucinous adenocarcinoma & $4(9)$ & $1(4)$ & & $5(8)$ & $0(0)$ & \\
\hline Pathological lymphatic invasion & & & 0.028 & & & 0.759 \\
\hline Negative & $30(65)$ & $10(37)$ & & $34(56)$ & $6(50)$ & \\
\hline Positive & $16(35)$ & $17(63)$ & & $27(44)$ & $6(50)$ & \\
\hline Pathological vessel invasion & & & 0.623 & & & $>0.999$ \\
\hline Negative & $44(96)$ & $25(93)$ & & $57(93)$ & $12(100)$ & \\
\hline Positive & $2(4)$ & $2(7)$ & & $4(7)$ & $0(0)$ & \\
\hline Pathological T status & & & 0.041 & & & 0.211 \\
\hline $1 \mathrm{a}$ & $12(26)$ & $1(4)$ & & $13(21)$ & $0(0)$ & \\
\hline $1 b$ & $24(52)$ & $16(59)$ & & $32(53)$ & $8(67)$ & \\
\hline $1 \mathrm{c}$ & $10(22)$ & $10(37)$ & & $16(26)$ & $4(33)$ & \\
\hline Pathological N status & & & $<0.001$ & & & 0.987 \\
\hline Negative & $42(91)$ & $12(44)$ & & $45(74)$ & $9(75)$ & \\
\hline $\mathrm{n} 1$ & $1(2)$ & $6(22)$ & & $6(10)$ & $1(8)$ & \\
\hline $\mathrm{n} 2$ & $3(7)$ & $9(33)$ & & $10(16)$ & $2(17)$ & \\
\hline Periostin expression & & & 0.003 & & & $>0.999$ \\
\hline Low & $31(67)$ & $8(30)$ & & $33(54)$ & $6(50)$ & \\
\hline High & $15(33)$ & $19(70)$ & & $28(46)$ & $6(50)$ & \\
\hline
\end{tabular}

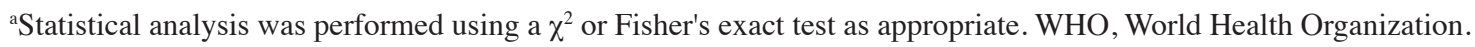

multivariate Cox proportional hazards regression models. All variables identified as statistically significant were included in the multivariate model. The Kaplan-Meier method was used to estimate time to recurrence or death. In addition, the cumulative incidence of recurrence or survival according to the risk factors assessed by multivariate analysis were also calculated and analyzed using the log-rank test. $\mathrm{P}<0.05$ was considered to indicate a statistically significant difference. All statistical analyses were performed using IBM SPSS Statistics, version 23 (IBM Corp.).

\section{Results}

Patient characteristics and pathological findings of resected specimen. The median age of the patients was 66 years (range, 45-85 years). Detailed clinicopathological characteristics of the patients are provided in Table I. During the follow-up period (median follow-up, 77 months; range, 11-218 months), recurrence occurred in 27 patients, and 46 patients had no evidence of recurrence. A total of 12 patients died following tumor recurrence. The interval between removal and recurrence ranged from 5-94 months (median, 18 months), and the interval until death ranged from 11-199 months (median, 54 months).

Among patient age, sex and pathological findings, statistically significant differences were identified between patients with and without recurrence based on histological typing $(\mathrm{P}=0.003)$, pathological lymphatic invasion $(\mathrm{P}=0.028)$, T status $(\mathrm{P}=0.041), \mathrm{N}$ status $(\mathrm{P}<0.001)$ and periostin expression $(\mathrm{P}=0.003)$ (Table I). Within the histological subtypes, patients with the acinar predominant type exhibited more instances of recurrence (11 cases of recurrence and 3 cases without recurrence), and patients with the papillary predominant type 
Table II. Comparison of CT parameters between the no recurrence $(n=46)$ and recurrence $(n=27)$ groups, and between the survival $(\mathrm{n}=61)$ and death $(\mathrm{n}=12)$ groups.

\begin{tabular}{|c|c|c|c|c|c|c|c|}
\hline Parameters & $\begin{array}{l}\text { Interobserver } \\
\text { agreements, } k \text { value } \\
\text { (lower to upper } \\
95 \% \text { confidence } \\
\text { intervals) }\end{array}$ & $\begin{array}{c}\text { No recurrence, } \\
\mathrm{n}(\%)\end{array}$ & $\begin{array}{l}\text { Recurrence, } \\
\mathrm{n}(\%)\end{array}$ & P-value ${ }^{b}$ & $\begin{array}{l}\text { Survival, } \mathrm{n} \\
(\%)\end{array}$ & $\begin{array}{l}\text { Death, } \mathrm{n} \\
(\%)\end{array}$ & P-value ${ }^{b}$ \\
\hline Contour & $0.64(0.30-0.98)$ & & & 0.623 & & & 0.521 \\
\hline Smooth & & $2(4)$ & $2(7)$ & & $3(5)$ & $1(8)$ & \\
\hline Irregular & & $44(96)$ & $25(93)$ & & $58(95)$ & $11(92)$ & \\
\hline Type of nodule & $0.83(0.71-0.96)$ & & & 0.057 & & & 0.533 \\
\hline Part-solid GGN & & $28(61)$ & $10(37)$ & & $33(54)$ & $5(42)$ & \\
\hline Solid nodule & & $18(39)$ & $17(53)$ & & $28(46)$ & $7(58)$ & \\
\hline CT solid score, $\%$ & $0.71(0.52-0.90)$ & & & 0.001 & & & 0.089 \\
\hline$\leq 80$ & & $21(46)$ & $2(7)$ & & $22(36)$ & $1(8)$ & \\
\hline$>80$ & & $25(54)$ & $25(93)$ & & $39(64)$ & $11(92)$ & \\
\hline $\begin{array}{l}\text { Air-bronchogram and/or } \\
\text { bubble-like lucencies }\end{array}$ & $0.85(0.73-0.97)$ & & & 0.085 & & & 0.720 \\
\hline Absence & & $32(70)$ & $24(89)$ & & $46(75)$ & $10(83)$ & \\
\hline Presence & & $14(30)$ & $3(11)$ & & $15(25)$ & $2(17)$ & \\
\hline Involved vessels, $\mathrm{n}$ & $0.70(0.53-0.86)$ & & & 0.331 & & & 0.124 \\
\hline$<2$ & & $27(59)$ & $12(44)$ & & $30(49)$ & $9(75)$ & \\
\hline$\geq 2$ & & $19(41)$ & $15(56)$ & & $31(51)$ & $3(25)$ & \\
\hline Linear strands (shape) & $0.75(0.60-0.90)$ & & & $>0.999$ & & & 0.761 \\
\hline Type 1 (none or fine) & & $25(54)$ & $14(52)$ & & $32(53)$ & $7(58)$ & \\
\hline $\begin{array}{l}\text { Type } 2 \text { (Coarse or } \\
\text { triangular) }\end{array}$ & & $21(46)$ & $13(48)$ & & $29(47)$ & $5(42)$ & \\
\hline Linear strands, $\mathrm{n}$ & $0.68(0.48-0.89)$ & & & 0.532 & & & 0.438 \\
\hline$<2$ & & $39(85)$ & $21(78)$ & & $51(84)$ & $9(75)$ & \\
\hline$\geq 2$ & & $7(15)$ & $6(22)$ & & $10(16)$ & $3(25)$ & \\
\hline
\end{tabular}

${ }^{\mathrm{a}}$ Agreements of two independent observers were calculated with $\kappa$ statistics. ${ }^{\mathrm{b}}$ Statistical analysis for categorical data was performed using a $\chi^{2}$ or Fisher's exact test as appropriate. GGN, ground-glass nodule.

exhibited fewer cases of recurrence (6 cases of recurrence and 28 cases of no recurrence; Table I). In the micropapillary type, which is associated with a poor prognosis in general, recurrence occurred in one-third of patients ( 2 out of 6 patients). However, univariate analysis with Cox proportional hazards regression models revealed no significant difference between the histological subtype and recurrence.

$C T$ parameters prior to surgery. There was a substantial to almost perfect agreement between the two observers in terms of the classification of the seven thin-section $\mathrm{CT}$ parameters $(\kappa=0.64-0.85$; Table II). Among the CT parameters, only the CT solid score was significantly correlated with postoperative recurrence $(\mathrm{P}=0.001$; Table $\mathrm{II})$. ROC analysis was performed to define the appropriate threshold of the CT solid score. The area under the curve (AUC) for predicting recurrence was 0.70 [95\% confidence interval (CI), 0.58-0.82] and the AUC for predicting death was 0.62 (95\% CI, 0.48-0.77). Both AUC gave the highest sensitivity and specificity when the threshold of the CT solid score was $80 \%$. Recurrence occurred in $50 \%$
(25 out of 50 patients) of patients with a CT solid score $>80 \%$, compared with only $9 \%$ ( 2 out of 23 patients) of those with a CT solid score $\leq 80 \%$.

Univariate analysis using the Cox proportional hazards model for recurrence (Table III) found that pathological lymphatic invasion [hazard ratio (HR), 3.16; 95\% CI, 1.40-7.14; $\mathrm{P}=0.006]$, pathological T1b vs. T1a (HR, 7.93; 95\% CI, 1.01-62.50; $\mathrm{P}=0.048$ ), pathological N1 vs. N0 (HR, 4.26; $95 \% \mathrm{CI}, 1.79-10.10 ; \mathrm{P}=0.001), \mathrm{CT}$ solid score $>80$ vs. $\leq 80 \%$ (HR, 6.85; 95\% CI, 1.62-29.41; $\mathrm{P}=0.009)$, and high periostin expression vs. low (HR, 3.46; 95\% CI, 1.51-7.94; $\mathrm{P}=0.003$ ) were significant predictors of recurrence. In the multivariate analysis (Table IV), a CT solid score $>80 \%$ (HR, 10.10; 95\% CI, 2.29-45.46; $\mathrm{P}=0.002)$ and high periostin expression (HR, 3.72; 95\% CI, 1.35-10.31; $\mathrm{P}=0.011$ ) remained significant for recurrence.

Combined analysis of the CT solid score and periostin expression. The cumulative incidence of recurrence according to the $\mathrm{CT}$ solid score and periostin expression was 
Table III. Univariate analysis using the Cox proportional hazards model for recurrence and overall survival.

\begin{tabular}{|c|c|c|c|c|c|c|c|c|}
\hline \multirow[b]{3}{*}{ Explanatory variables } & \multicolumn{4}{|c|}{ Recurrence } & \multicolumn{4}{|c|}{ Overall survival } \\
\hline & \multirow[b]{2}{*}{ HR } & \multicolumn{2}{|c|}{$95 \% \mathrm{CI}$} & \multirow[b]{2}{*}{ P-value } & \multirow[b]{2}{*}{ HR } & \multicolumn{2}{|c|}{$95 \% \mathrm{CI}$} & \multirow[b]{2}{*}{ P-value } \\
\hline & & Lower & Upper & & & Lower & Upper & \\
\hline Age at surgery ( $>65$ vs. $\leq 65$ years $)$ & 2.08 & 0.95 & 4.54 & 0.066 & 2.24 & 0.68 & 7.45 & 0.187 \\
\hline Sex (male vs. female) & 1.06 & 0.49 & 2.28 & 0.883 & 1.10 & 0.35 & 3.41 & 0.870 \\
\hline \multicolumn{9}{|l|}{ Histological typing (vs. lepidic predominant) } \\
\hline Acinar predominant & 3.60 & 0.37 & 34.98 & 0.270 & 0.51 & 0.11 & 2.32 & 0.382 \\
\hline Papillary predominant & 7.44 & 0.95 & 58.09 & 0.056 & 0.64 & 0.17 & 2.37 & 0.506 \\
\hline Micropapillary predominant & 1.48 & 0.18 & 12.51 & 0.718 & 4.20 & 0.92 & 19.40 & 0.065 \\
\hline Solid predominant with mucin production & 2.38 & 0.22 & 26.35 & 0.479 & 0.49 & 0.11 & 2.24 & 0.355 \\
\hline Invasive mucinous adenocarcinoma & 4.82 & 0.53 & 43.52 & 0.161 & 0.59 & 0.20 & 1.71 & 0.334 \\
\hline Pathological lymphatic invasion (presence vs. absence) & 3.16 & 1.40 & 7.14 & 0.006 & 1.53 & 0.48 & 4.80 & 0.468 \\
\hline Pathological vessel invasion (presence vs. absence) & 1.12 & 0.26 & 4.81 & 0.879 & 22.00 & 0.00 & 566.44 & 0.611 \\
\hline \multicolumn{9}{|l|}{ Pathological T status (vs. T1a) } \\
\hline $\mathrm{T} 1 \mathrm{~b}$ & 7.93 & 1.01 & 62.50 & 0.048 & 1.53 & 0.46 & 5.09 & 0.488 \\
\hline $\mathrm{T} 1 \mathrm{c}$ & 1.44 & 0.65 & 3.19 & 0.376 & 1.07 & 0.32 & 3.57 & 0.907 \\
\hline \multicolumn{9}{|l|}{ Pathological N status (vs. N0) } \\
\hline $\mathrm{N} 1$ & 4.26 & 1.79 & 10.10 & 0.001 & 1.30 & 0.28 & 5.88 & 0.772 \\
\hline $\mathrm{N} 2$ & 1.14 & 0.40 & 3.23 & 0.808 & 1.39 & 0.13 & 14.29 & 0.722 \\
\hline Contour (irregular vs. smooth) & 1.20 & 0.28 & 5.07 & 0.805 & 1.47 & 0.19 & 11.36 & 0.715 \\
\hline Part-solid GGN (vs. solid nodule) & 0.56 & 0.25 & 1.22 & 0.138 & 0.63 & 0.20 & 1.99 & 0.433 \\
\hline CT solid score (>80 vs. $\leq 80 \%$ ) & 6.85 & 1.62 & 29.41 & 0.009 & 6.20 & 0.80 & 47.61 & 0.081 \\
\hline $\begin{array}{l}\text { Air-bronchogram and/or bubble-like lucencies } \\
\text { (presence vs. absence) }\end{array}$ & 2.55 & 0.77 & 8.49 & 0.126 & 1.81 & 0.39 & 8.29 & 0.419 \\
\hline Involved vessels, $\mathrm{n}(\geq 2$ vs. $<2)$ & 1.44 & 0.67 & 3.13 & 0.346 & 2.58 & 0.69 & 9.55 & 0.156 \\
\hline $\begin{array}{l}\text { Linear strands shape [type } 2 \text { (coarse or triangular) vs. } \\
\text { type } 1 \text { (none or fine)] }\end{array}$ & 1.36 & 0.64 & 2.90 & 0.425 & 1.03 & 0.33 & 3.25 & 0.960 \\
\hline Linear strands, $\mathrm{n}(\geq 2$ vs. $<2)$ & 1.74 & 0.69 & 4.33 & 0.236 & 0.59 & 0.16 & 2.17 & 0.423 \\
\hline Periostin expression (high vs. low) & 3.46 & 1.51 & 7.94 & 0.003 & 0.69 & 0.22 & 2.14 & 0.517 \\
\hline
\end{tabular}

95\% CI, 95\% confidence interval; GGN, ground-glass nodule; HR, hazard ratio.

Table IV. Multivariate analysis using the Cox proportional hazards model for recurrence.

\begin{tabular}{lrrr}
\hline & \multicolumn{2}{c}{ Recurrence } \\
\cline { 2 - 4 } & & \multicolumn{2}{c}{$95 \%$ CI } \\
\cline { 3 - 4 } Explanatory variables & HR & Lower & Upper \\
\hline CT solid score (>80 vs. $\leq 80 \%)$ & 10.10 & 2.29 & 45.46 \\
Periostin expression (high vs. low) & 3.72 & 1.35 & 10.31 \\
\hline
\end{tabular}

95\% CI, 95\% confidence interval; HR, hazard ratio.

calculated (Fig. 4A and B). The cumulative incidence of recurrence was higher in patients with a CT solid score $>80 \%$ and high periostin expression compared with patients with a CT solid score $\leq 80 \%$ and low periostin expression. Furthermore, the cumulative recurrence rates among the three groups (both negative, CT solid score $>80 \%$ or high periostin expression, and both positive) were significantly different (log-rank, $\mathrm{P}<0.001$; Fig. 4C). On the other hand, univariate analysis using the Cox proportional hazards model for OS did not reveal any significant levels of association with any of the evaluated factors. 

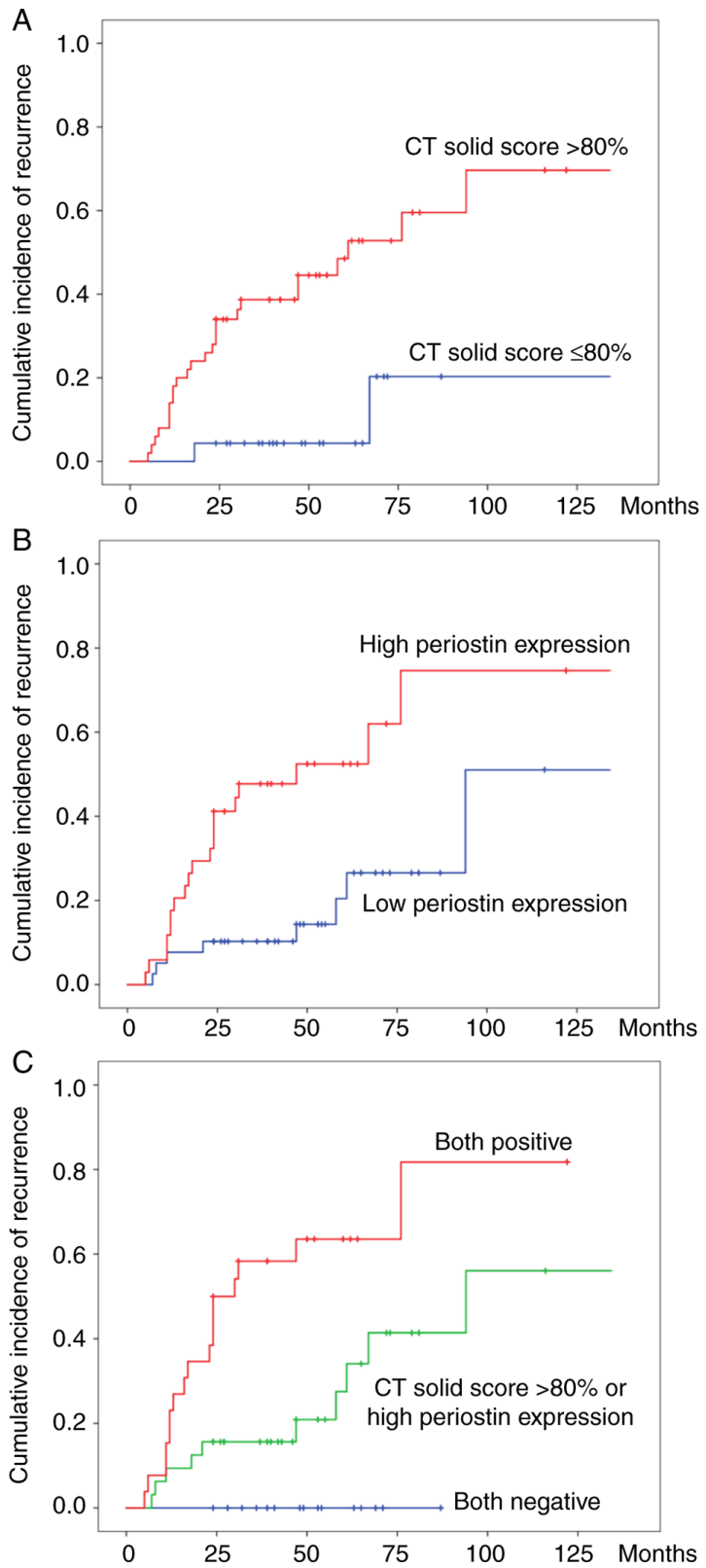

Figure 4. Cumulative incidence of recurrence based on the CT solid score, periostin expression and the combination of these. (A) Graph showing that the cumulative incidence of recurrence according to CT solid score on preoperative $\mathrm{CT}$ was higher in patients with solid score $>80 \%$ than in those with solid score $\leq 80 \%$ (log rank, $\mathrm{P}=0.002$ ). (B) Graph showing that the cumulative incidence of recurrence according to periostin expression was higher in patients with high expression than in those with low expression (log rank, $\mathrm{P}=0.002$ ). (C) Graph showing that the cumulative incidence of recurrence among three groups (both negative, CT solid score $>80 \%$ or high periostin expression, and both positive) was significantly different (log rank, $\mathrm{P}<0.001)$.

\section{Discussion}

Various biomarkers for predicting the prognosis of patients following surgical resection have been suggested, particularly for early-stage lung adenocarcinoma, which possibly includes poorer prognostic cases. Therefore, the aim of the present study was to search for potential alternative predictive factors, in addition to the preoperative CT finding. In the present study, it has been shown that the combined analysis of CT finding (the CT solid score) and pathological feature (periostin expression) could predict the likelihood of tumor recurrence more precisely than working on the basis of the CT findings only. The cumulative recurrence rates among the three groups (both negative, CT solid score $>80 \%$ or high periostin expression, and both positive) were significantly different (log-rank, $\mathrm{P}<0.001$; Fig. 4C). Notably, the cumulative recurrence rate of the 'both-negative' group was revealed to be zero. Therefore, even though CT reveals the presence of a solid component in $\mathrm{T} 1$ adenocarcinoma, when the solid component is $\leq 80 \%$ on thin-section CT and the periostin expression is weak in the pathological specimen, the risk of recurrence is very low. These results may contribute towards the provision of a more personalized treatment schedule, even in cases with the same $\mathrm{T} 1$ invasive adenocarcinoma.

As an imaging biomarker, previous studies have suggested that the solid component of the tumor on thin-section CT scans is associated with tumor invasiveness $(20,24,32)$. Present $\mathrm{T}$ status also gives great weight to solid component during tumor evaluation, therefore the value of the $\mathrm{CT}$ solid score of the tumor on preoperative CT scans was evaluated. Asamura et al (33) reported that the consolidation/tumor ratio (CTR) on preoperative CT could be used to predict survival outcomes in patients with non-invasive adenocarcinoma of the lung. They reported that T1 tumors with a smaller CTR had radiographically excellent prognostic characteristics and may show non-invasive adenocarcinomas. However, their study included pathologically non-invasive cases, which may have a more favorable prognosis. Among adenocarcinomas, the prognosis of AIS and MIA is generally favorable. These types of tumors predominantly consist of GGO on CT images, and several studies have revealed that the extent of GGO is a favorable prognostic factor, as GGO represents the less invasive component of adenocarcinoma as 'lepidic' growth. Therefore, the present study was limited to the assessment of invasive adenocarcinomas.

As a pathological biomarker, periostin expression was examined using immunohistological staining. A previous study showed that periostin acts as a ligand of integrin, which is a cell-surface receptor that activates the PI3-K/Akt pathway and promotes cancer cell survival, epithelial-mesenchymal transition, invasion and metastasis (13). Sasaki et al (15) suggested that periostin could be involved in cell adhesion and invasion in vitro. Murakami et al (34) investigated periostin expression quantitatively in NSCLC via immunohistochemical analysis, and they demonstrated that intra-tumoral periostin expression was an independent prognostic factor in NSCLC. Therefore, in the present study a combined analysis of the CT finding (i.e., the CT solid score) and pathological feature (periostin expression) was performed to determine whether the analysis could assist in predicting the prognosis more precisely than relying on CT finding only.

However, no predictors of OS were identified in the present study. Due to the various chemotherapeutic regimens with or without inclusion of immune checkpoint inhibitors that have been recently introduced to increase OS and/or progression-free survival, the patients with recurrence exhibited relatively long-term survival in the present study. It is assumed that the progression of salvage therapy contributed to extending OS in the cases of recurrence.

The present study has some limitations. The data were obtained from a single-center, the study was retrospective, and the cohort size was small. Moreover pre-operative CT 
was performed with a range of scanners and the performance changed notably with time. Thus, the accuracy of the morphological evaluation of the tumors on CT may vary from patient to patient. Additionally, there was no general consensus available on the optimal method for evaluating the solid component of the tumor. The solid component may have an irregular or scattered shape, and measurements may not be reproducible. Although the agreements of the two independent observers on the seven CT parameters ranged from good to excellent, the results may have been more precise if the size of the solid component and the whole nodule were measured using automated volumetric software. Finally, histopathological specimens were assessed only using hematoxylin and eosin staining, and immunohistochemical staining for periostin; other factors, such as EGFR mutants, were not assessed.

In conclusion, the combined analysis of the CT solid score before surgical resection and periostin expression in the resected specimen may be predictors of an increased likelihood of tumor recurrence, even in patients with T1 invasive adenocarcinoma. Excessive follow-up could be avoided in patients with low risk of recurrence. Shorter follow-up intervals should be recommended in patients with high risk of recurrence, even in T1 adenocarcinoma of the lung. In the latter group, recurrence may be identified earlier relative to the normal follow-up group, and prompt introduction of salvage treatment could increase the survival rate, even in recurrent cases.

\section{Acknowledgements}

Not applicable.

\section{Funding}

No funding was received.

\section{Availability of data and materials}

The datasets used and/or analyzed during the current study are available from the corresponding author on reasonable request.

\section{Authors' contributions}

RI and KF designed the study. STan, SN, KT, JF, KO, SO and KI made substantial contributions to the conception of the study. KF and TA supervised the study design. RI, SN, MK, AS, TC, TA, DM, STak, HI, KO, SO and KI acquired the data. RI, SN, KT, JF, MK, AS, TC, HI, KO, SO and KI confirmed the authenticity of the raw data. RI, STan, KT, JF, KO and $\mathrm{KF}$ analyzed and interpreted the data. RI and KF drafted the manuscript. RI, STan, KI and KF revised the manuscript critically for important intellectual content. All authors read and approved the final manuscript, and all authors provided their final approval of the version to be published.

\section{Ethics approval and consent to participate}

The Ethics Committee of Kurume University (Kurume, Japan) approved this retrospective study and waived the requirement for informed consent (approval no. 09113).

\section{Patient consent for publication}

Not applicable.

\section{Competing interests}

The authors declare that they have no competing interests.

\section{References}

1. Lewis DR, Check DP, Caporaso NE, Travis WD and Devesa SS: US lung cancer trends by histologic type. Cancer 120: 2883-2892, 2014

2. Travis WD, Brambilla E, Noguchi M, Nicholson AG, Geisinger KR, Yatabe Y, Beer DG, Powell CA, Riely GJ, Van Schil PE, et al: International association for the study of lung cancer/american thoracic society/european respiratory society international multidisciplinary classification of lung adenocarcinoma. J Thorac Oncol 6: 244-285, 2011.

3. Yoshizawa A, Motoi N, Riely GJ, Sima CS, Gerald WL, Kris MG, Park BJ, Rusch VW and Travis WD: Impact of proposed IASLC/ATS/ERS classification of lung adenocarcinoma: Prognostic subgroups and implications for further revision of staging based on analysis of 514 Stage I cases. Mod Pathol 24: 653-664, 2011.

4. Lee HY, Jeong JY, Lee KS, Kim HJ, Han J, Kim BT, Kim J, Shim YM, Kim JH and Song I: Solitary pulmonary nodular lung adenocarcinoma: Correlation of histopathologic scoring and patient survival with imaging biomarkers. Radiology 264 : 884-893, 2012.

5. Kadota K, Villena-Vargas J, Yoshizawa A, Motoi N, Sima CS, Riely GJ, Rusch VW, Adusumilli PS and Travis WD: Prognostic significance of adenocarcinoma in situ, minimally invasive adenocarcinoma, and nonmucinous lepidic predominant invasive adenocarcinoma of the lung in patients with stage I disease. Am J Surg Pathol 38: 448-460, 2014.

6. Edge SB and Compton CC: The American joint committee on cancer: The 7th edition of the AJCC cancer staging manual and the future of TNM. Ann Surg Oncol 17: 1471-1474, 2010.

7. Chae HD, Park CM, Park SJ, Lee SM, Kim KG and Goo JM: Computerized texture analysis of persistent part-solid ground-glass nodules: Differentiation of preinvasive lesions from invasive pulmonary adenocarcinomas. Radiology 273: 285-293, 2014.

8. Heidinger BH, Anderson KR, Nemec U,Costa DB, Gangadharan SP, VanderLaan PA and Bankier AA: Lung adenocarcinoma manifesting as pure ground-glass nodules: Correlating CT size, volume, density, and roundness with histopathologic invasion and size. J Thorac Oncol 12: 1288-1298, 2017.

9. Takeshita S, Kikuno R, Tezuka K and Amann E: Osteoblastspecific factor 2: Cloning of a putative bone adhesion protein with homology with the insect protein fasciclin I. Biochem J 294: 271-278, 1993 .

10. Kudo Y, Siriwardena BS, Hatano H, Ogawa I and Takata T: Periostin: Novel diagnostic and therapeutic target for cancer. Histol histopathol 22: 1167-1174, 2007.

11. Okamoto M, Hoshino T, Kitasato Y, Sakazaki Y, Kawayama T, Fujimoto K, Ohshima K, Shiraishi H, Uchida M, Ono J, et al: Periostin, a matrix protein, is a novel biomarker for idiopathic interstitial pneumonias. Eur Respir J 37: 1119-1127, 2011.

12. Conway SJ, Izuhara K, Kudo Y, Litvin J, Markwald R, Ouyang G, Arron JR, Holweg CT and Kudo A: The role of periostin in tissue remodeling across health and disease. Cell Mol Life Sci 71: 1279-1288, 2014

13. Ruan K, Bao S and Ouyang G: The multifaceted role of periostin in tumorigenesis. Cell Mol Life Sci 66: 2219-2230, 2009.

14. Sasaki H, Dai M, Auclair D, Kaji M, Fukai I, Kiriyama M, Yamakawa Y, Fujii Y and Chen LB: Serum level of the periostin, a homologue of an insect cell adhesion molecule, in thymoma patients. Cancer Lett 172: 37-42, 2001.

15. Sasaki H, Dai M, Auclair D, Fukai I, Kiriyama M, Yamakawa Y, Fujii Y and Chen LB: Serum level of the periostin, a homologue of an insect cell adhesion molecule, as a prognostic marker in nonsmall cell lung carcinomas. Cancer 92: 843-848, 2001.

16. Fujimoto K, Kawaguchi T, Nakashima O, Ono J, Ohta S, Kawaguchi A, Tonan T, Ohshima K, Yano H, Hayabuchi N, et al: Periostin, a matrix protein, has potential as a novel serodiagnostic marker for cholangiocarcinoma. Oncol Rep 25: 1211-1216, 2011. 
17. Kudo Y, Ogawa I, Kitajima S, Kitagawa M,Kawai H, Gaffney PM, Miyauchi $\mathrm{M}$ and Takata T: Periostin promotes invasion and anchorage-independent growth in the metastatic process of head and neck cancer. Cancer Res 66: 6928-6935, 2006.

18. Fukushima N, Kikuchi Y, Nishiyama T, kudo A and Fukayama M: Periostin deposition in the stroma of invasive and intraductal neoplasms of the pancreas. Modern Pathol 21: 1044-1053, 2008.

19. Hong LZ, Wei XW, Chen JF and Shi Y: Overexpression of periostin predicts poor prognosis in non-small cell lung cancer. Oncol Let 6: 1595-1603, 2013.

20. Kim EA, Johkoh T, Lee KS, Han J, Fujimoto K, Sadohara J, Yang PS, Kozuka T, Honda O and Kim S: Quantification of ground-glass opacity on high-resolution CT of small peripheral adenocarcinoma of the lung: Pathologic and prognostic implications. Am J Roentgenol 177: 1417-1422, 2001.

21. Maeyashiki T, Suzuki K, Hattori A, Matsunaga T, Takamochi K and Oh S: The size of consolidation on thin-section computed tomography is a better predictor of survival than the maximum tumor dimension in resectable lung cancer. Eur J Cardiothorac Surg 43: 915-918, 2013.

22. Burt BM, Leung AN, Yanagawa M, Chen W, Groth SS, Hoang CD, Nair VS and Shrager JB: Diameter of solid tumor component alone should be used to establish $\mathrm{T}$ stage in lung adenocarcinoma. Ann Surg Oncol 22 (Suppl 3): S1318-S1323, 2015.

23. Shikuma K, Menju T, Chen F, Kubo T, Muro S, Sumiyoshi S, Ohata K, Sowa T, Nakanishi T, Cho H, et al: Is volumetric 3-dimensional computed tomography useful to predict histological tumor invasiveness? Analysis of 211 lesions of cT1N0M0 lung adenocarcinoma. Interact Cardiovasc Thorac Surg 22: 831-838, 2016

24. Hattori A, Matsunaga T, Hayashi T, Takamochi K, Oh S and Suzuki K: Prognostic impact of the findings on thin-section computed tomography in patients with subcentimeter non-small cell lung cancer. J Thorac Oncol 12: 954-962, 2017.

25. International Association for the Study of Lung Cancer: Staging Manual in Thoracic Oncology. 2nd edition. Editorial Rx Press, North Fort Myers, FL, 2016.
26. Ginsberg RJ and Rubinstein LV: Randomized trial of lobectomy versus limited resection for T1 N0 non-small cell lung cancer. Ann Thoracic Surg 60: 615-623, 1995.

27. Bankier AA, MacMahon H, Goo JM, Rubin GD, SchaeferProkop CM and Naidich DP: Recommendations for measuring pulmonary nodules at CT: A statement from the Fleischner Society. Radiology 285: 584-600, 2017.

28. Hansell DM, Bankier AA, MacMahon H, McLoud TC, Müller NL and Remy J: Fleischner society: Glossary of terms for thoracic imaging. Radiology 246: 697-722, 2008.

29. Zwirewich CV, Vedal S, Miller RR and Müller NL: Solitary pulmonary nodule: High-resolution CT and radiologic-pathologic correlation. Radiology 179: 469-476, 1991.

30. Li M, Wang Y, Chen Y and Zhang Z: Identification of preoperative prediction factors of tumor subtypes for patients with solitary ground-glass opacity pulmonary nodules. J Cardiothorac Surg 13: 9, 2018.

31. Kundel HL and Polansky M: Measurement of observer agreement. Radiology 228: 303-308, 2003.

32. Miao Y, Zhang J, Zou J, Zhu Q, Lv T and Song Y: Correlation in histological subtypes with high resolution computed tomography signatures of early stage lung adenocarcinoma. Transl Lung Cancer Res 6: 14-22, 2017.

33. Asamura H, Hishida T, Suzuki K, Koike T, Nakamura K, Kusumoto M, Nagai K, Tada H, Mitsudomi T, Tsuboi M, et al: Radiographically determined noninvasive adenocarcinoma of the lung: Survival outcomes of Japan clinical oncology group 0201. J Thorac Cardiovasc Surg 146: 24-30, 2013.

34. Murakami D, Takamori S, Kawahara A,Mitsuoka M,Kashihara M, Yoshiyama K, Matsumoto R, Yokoyama S, Fujimoto K, Kawaguchi A, et al: Periostin expression in non-small cell lung cancer: Clinical significance. Kurume Med J 64: 13-20, 2017.

This work is licensed under a Creative Commons Attribution-NonCommercial-NoDerivatives 4.0 International (CC BY-NC-ND 4.0) License. 